\title{
Effects of solute content on grain refinement in an isothermal melt
}

\author{
H. Men and Z. Fan ${ }^{*}$ \\ The EPSRC Centre for Innovative Manufacturing in Liquid Metal Engineering, BCAST (Brunel \\ Centre for Advanced Solidification Technology), Brunel University, Uxbridge, Middlesex, UB8 \\ 3PH, UK.
}

\begin{abstract}
It is well accepted in the literature that for effective grain refinement some solute is required in the melt to restrict the growth of the solid even if potent nucleating particles with a favourable physical nature are present. In this paper, we investigate the effect of solute on grain initiation in an isothermal melt, and an analytical model is developed to account for the effect of solute elements on the grain size. This study revealed that the solute elements in the liquid ahead of the growing crystals reduce the growth velocity of the nucleated crystals, and increase the maximum undercooling achievable before recalescence. This allows for more particles to be active for nucleation, and consequently increases the number density of active particles, giving rise to a finer grain size. The analytical model shows that the final grain size can be related to the maximum undercooling, average growth velocity and solid fraction at the moment of the recalescence. Further analysis using the free growth model and experimental data in the literature revealed that for a given alloy system solidified under similar conditions the grain size can be empirically related to $1 / Q$ ( $Q$ is the growth restriction factor) to a power of $1 / 3$, which is considerably different from the empirical linear relationship in the literature. It is demonstrated that the $1 / 3$ power law can describe the experimental data more accurately than a linear relationship.
\end{abstract}

Keywords: Solidification; Grain refinement; Undercooling; Isothermal melt; Solute elements

\section{Introduction}

The solute content has significant effects on grain refinement of engineering alloys [1-4]. For effective refinement, not only do the grain refining particles themselves need to be potent for heterogeneous nucleation, but also some solute elements are required to enhance the efficiency of the particles by restricting the growth of the solid, either at a columnar growth front competing with equiaxed solidification or from particles where nucleation has already occurred [5-7]. The degree of growth restriction for a particular solute was initially described by the constitutional-supercooling parameter $P[8]$ :

$$
P=\frac{m(k-1) C_{0}}{k}
$$

where $m$ is the liquidus slope, $k$ the equilibrium partition coefficient, and $C_{0}$ the solute content in the alloy melt. Maxwell and Hellawell [9] suggested that, for spherical growth restricted by the partitioning of a single solute, to a good approximation the crystal growth rate for a given undercooling is inversely proportional to the parameter $Q$ :

$$
Q=C_{0} m(k-1)
$$

The parameter $Q$ is usually referred to as the growth restriction factor, and is used as a measure of the effects of solute on grain refinement in the absence of solute interactions [10-16]. It was found

\footnotetext{
* Corresponding author at: BCAST (Brunel Centre for Advanced Solidification Technology), Brunel University, Uxbridge, Middlesex UB8 3PH, UK. Tel.: +44 1895 266406; fax: +44 1895269758.

E-mail address: Zhongyun.Fan@brunel.ac.uk (Z. Fan).
} 
that the experimental data were better interpreted in terms of $Q$ [17]. Empirically, the average grain size $\bar{l}$ can be described as a linear function of $1 / Q$ for various $\mathrm{Al}[4,10-14,18,19], \mathrm{Mg}[1,20]$ and $\mathrm{Ti}$ alloys [21]:

$$
\bar{l}=a+b / Q
$$

where $a$ and $b$ are empirical constants. By further analysis of the experimental data, it is believed that $a$ is related to the number density of active nucleating particles and $b$ is related to the efficiency of the nucleating particles [22].

To characterize and quantify the effect of nucleating particles on grain refinement, the contribution made by solute elements needs to be established. Maxwell and Hellawell [9] analysed the heterogeneous nucleation in a spatially isothermal melt using the classical heterogeneous nucleation theory by assuming spherical diffusion-controlled growth. They demonstrated that the latent heat evolved by the growth of the solid raises the melt temperature above the heterogeneous nucleation temperature (recalescence), and thus suppresses further nucleation events. The role of the solute is to restrict the growth rate of the growing interface, which, in turn, allows time for further nucleation events to occur, and the final grain size is the result of competition between heterogeneous nucleation and growth.

Winegard and Chalmers [23] proposed the constitutional supercooling driven nucleation model, and suggested that the constitutional supercooling arising from solute enrichment in the liquid ahead of an advancing solid front could trigger nucleation on available nucleating particles. Following this nucleation mechanism, StJohn and co-workers [14,24] considered grain nucleation in the liquid ahead of a growing crystal with a small temperature gradient. They suggested that nucleation occurs when the amount of constitutional supercooling generated, $\Delta T_{\mathrm{cs}}$, reaches the undercooling required for nucleation, $\Delta T_{\mathrm{n}}$, and that the constitutional supercooling driven nucleation mechanism is responsible for the development of the solidified grain structure. Such semi-empirical models $[14,24]$ were presented by a linear relationship between $\bar{l}$ and $1 / Q$. On the other hand, Quested and Greer [25] analysed the efficiency of grain refiners during directional solidification, and found that the diffusional composition profile from the growing grains actually reduced the undercooling available for grain initiation.

In the models mentioned previously, single-sized nucleating particles were assumed explicitly or implicitly. Greer et al. [17] found that the efficiency of a grain refiner could be explained better by using the free-growth criterion to describe grain initiation in the isothermal melt, in which a distribution of particle sizes was assumed. The undercooling required for grain initiation $\left(\Delta T_{\mathrm{fg}}\right)$ on an individual particle with size $d$ is given by [17]:

$$
\Delta T_{\mathrm{fg}}=\frac{4 \sigma}{\Delta S_{\mathrm{v}} d}
$$

where $\sigma$ is the solid-liquid interfacial energy and $\Delta S_{\mathrm{v}}$ the entropy of fusion per unit volume. The free growth model revealed that the larger particles become active at a smaller undercooling, and most of the smaller particles remain inactive due to the occurrence of recalescence. For small castings the assumption of an isothermal melt and the application of the free growth criterion give a quantitatively correct prediction of the grain size with a numerical calculation [17,26-28].

In this paper, we analyse the effect of solute content on grain refinement in an isothermal melt and the effect of constitutional undercooling on grain initiation. An analytical model will be presented to predict the grain size as a function of solute content in an isothermal melt. The analytical model will be validated against experimental results available in the literature.

\section{Effect of constitutional undercooling on grain initiation}


It is well known that a solidification structure depends strongly on the solidification conditions. For simplicity, we will confine our discussion in this paper to solidification under isothermal conditions. Isothermal solidification is not only a hypothetical condition, but is also found in many practical cases as a good approximation. Due to the large thermal diffusion coefficient in the liquid state, the thermal diffusion length is usually two to three orders of magnitude greater than the solute diffusion length [9]. This makes it possible to achieve isothermal or near-isothermal solidification through the interplay between the external heat extraction and the latent heat release from the growing solid phase [17,26]. Such cases can be found in solidification of small castings with a low cooling rate, solidification of alloys with a low superheat and formation of the equiaxed zoon in DC casting.

Fig. 1 shows a typical cooling curve during solidification under isothermal conditions. According to the free growth model [17], nucleation starts on the largest particle at a temperature just below the alloy liquidus $\left(T_{1}\right)$, and progressively on the smaller particles. Nucleation ceases when recalescence occurs at temperature $T_{\mathrm{f}}$, at which the external heat extraction balances the latent heat release due to the growth of the already nucleated crystals. $T_{\mathrm{f}}$ corresponds to the temperature for the smallest active particle to nucleate. The thermal plateau after recalescence corresponds to the growth temperature $\left(T_{\mathrm{g}}\right)$ of the nucleated crystals. The maximum nucleation undercooling $\left(\Delta T_{\mathrm{re}}\right)$ at recalescence can be approximated as the difference between $T_{\mathrm{g}}$ and $T_{\mathrm{f}}$. There should be no further nucleation events after $T_{\mathrm{f}}$, and further solidification can only be achieved by crystal growth. A typical feature of isothermal solidification is the existence of recalescence [17,26]. To analyse the effect of solute contents on grain initiation, we will focus our discussion on the temperature range between $T_{1}$ and $T_{\mathrm{f}}$.

Between $T_{1}$ and $T_{\mathrm{f}}$, there is a competition between nucleation of new crystals and growth of the existing crystals. The growth of an existing crystal will reject solute atoms from the solid into the liquid, resulting in an enrichment of solute in the liquid at the solid/liquid (S/L) interface if the partition coefficient $k$ is less than unity. Fig. 2 shows a schematic illustration of a growing crystal with a radius $r$ and a characteristic width $(D / V)$ of the solute concentration profile in the liquid ahead of the growing crystal, where $D$ is the diffusion coefficient of the solute in the liquid and $V$ is the crystal growth velocity. It is assumed that the solute atoms rejected by the growing crystal diffuse only into the region between $r$ and $r+D / V$. Fig. 3 shows a schematic representation of the concentration profile ahead of a growing crystal. $C_{\mathrm{s}}$ and $C_{\mathrm{i}}$ are the solute concentrations in the solid and liquid, respectively, at the $\mathrm{S} / \mathrm{L}$ interface. For simplicity, the solute content in the solid can be considered to be $C_{\mathrm{s}}=k C_{0}$, where $C_{0}$ is bulk alloy composition. At the initial stage of solidification, the solute profile in the liquid between $r$ and $r+D / V$ can be well approximated by a straight line:

$$
C \&=C_{\mathrm{i}}-\frac{C_{\mathrm{i}}-C_{0}}{D / V} x \text {. }
$$

Thus, $C_{\mathrm{i}}$ can be obtained through the equality between the amount of solute rejected from the growing solid and the excess solute accumulated in the region between $r$ and $r+D / V$. The constitutional (solute) undercooling, $\Delta T_{\mathrm{s}}$, ahead of the growing crystal is then given by:

$$
\Delta T_{\mathrm{s}}=m \boldsymbol{C}_{\mathrm{i}}-C_{0} \text { - }
$$

It is clear that both $C_{\mathrm{i}}$ and $\Delta T_{\mathrm{s}}$ increase with the crystal growth. Thus, $\Delta T_{\mathrm{s}}$ reaches a maximum value at $T_{\mathrm{f}}$, and therefore we only need to consider $\Delta T_{\mathrm{s}}$ at the moment of recalescence.

The key question to answer is whether constitutional undercooling generated by the rejected solute from the growing crystals can activate nucleation on new particles. The physical properties used in the calculation are listed in Table 1 . The calculated $C_{0}, C_{\mathrm{s}}, C_{\mathrm{i}}$ and $\Delta T_{\mathrm{s}}$ for various alloy compositions and process conditions are listed in Table 2. Fig. 4 shows the schematic liquidus $\left(T_{1}\right)$ and local temperature $\left(T_{\mathrm{lc}}\right)$ profiles ahead of a growing crystal. $\Delta T_{\mathrm{kc}}$ is the sum of the kinetic and curvature undercoolings. $\Delta T$ is the local thermal undercooling, $\Delta T=\Delta T_{\mathrm{re}}$ at the moment of recalescence. At a very early stage of solidification, $\Delta T_{\mathrm{s}}$ is usually much smaller than $\Delta T\left(\Delta T_{\mathrm{re}}\right)$, as 
indicated in Table 2. The liquidus in the region of $D / V$ ahead of the growing crystals is decreased, due to the increase of solute content. Thus, the diffusional composition ahead of a growing crystal actually reduces the undercooling available for grain initiation. However, this conclusion is drawn based on the data in Table 2, where the cooling rate is relatively small. To confirm this conclusion it is necessary to investigate the effect of cooling rate on solute undercooling.

Using the approach adopted in our previous study [28], $\bar{l}, \Delta T_{\mathrm{re}}$ and the solid fraction at recalescence $\left(f_{\text {sr }}\right)$ are obtained for an AZ91 alloy solidified in a large range of cooling rates $\left(0.1 \sim 10^{3}\right.$ $\mathrm{K} / \mathrm{s}$ ) with intensive melt shearing, and listed in Table 3. $C_{0}, C_{\mathrm{s}}, C_{\mathrm{i}}$ and $\Delta T_{\mathrm{s}}$ were calculated using the same procedure, and are also summarized in Table 3. The variation of $\Delta T_{\mathrm{s}}$ and $\Delta T_{\mathrm{re}}$ as a function of the cooling rate reveals that $\Delta T_{\text {re }}$ increases significantly with the cooling rate, indicating that at a higher cooling rate the smaller particles become active, resulting in an increase in the total number of active particles. Consequently, the grains become finer at a higher cooling rate. On the other hand, $\Delta T_{\mathrm{s}}$ decreases with an increase of the cooling rate, and becomes negligible when the cooling rate is above $1 \mathrm{~K} / \mathrm{s}$. Thus, at a high cooling rate the constitutional undercooling ahead of the growing crystals becomes negligible compared to $\Delta T_{\text {re, }}$ and could have little effect on grain initiation. It should be pointed out that such an analysis can only be treated qualitatively since the assumption of isothermal solidification becomes uncertain at a high cooling rate.

On the other hand, for the cases of a small cooling rate the latent heat released by the growing crystal could lead to a temperature rise at the $\mathrm{S} / \mathrm{L}$ interface, i.e. local recalescence. The heat flow from the $S / L$ interface into the liquid will result in a negative temperature gradient surrounding the growing crystal, and the thermal undercooling becomes smaller in the melt adjacent to the S/L interface [9]. Therefore, the local recalescence further reduces the available undercooling for grain initiation in the diffusional zone of a growing crystal, and outside this region there always is a larger local undercooling $(\Delta T)$ available for grain initiation.

Based on the above analysis, it can be safely concluded that in an isothermal melt, constitutional undercooling due to the growth of existing crystals cannot activate nucleation in an isothermal melt. This is in direct contrast to the recently proposed constitutional undercooling driven nucleation in the literature $[14,24]$. Further theoretical analysis is required to understand the exact mechanism of solute effects on heterogeneous nucleation.

\section{Modelling the solute effects on grain initiation}

\subsection{Basic considerations}

Solidification conditions determine the solidification structure for a given alloy composition. In this study we assume that solidification occurs in an isothermal melt. During solidification of an isothermal melt, the latent heat released by the growing crystals leads to recalescence, which marks the end of nucleation according to the free growth criterion. The growth of the nucleated crystals before recalescence is expected to be spherical. Mullins and Sekerka [34] examined the stability of spheres growing by diffusion of solute, and concluded that morphological instabilities will occur when the size of a sphere crystal is 7 times the critical nucleation radius. At the time of recalescence in an isothermal melt, $r$ and $f_{\text {sr }}$ are usually very small, with $f_{\text {sr }}$ being in the order of magnitude of $10^{-}$ 4 for typical $\mathrm{Al}[9,26]$ and $\mathrm{Mg}$ alloy melts [28]. For an isothermal melt solidifying in the temperature range between $T_{1}$ and $T_{\mathrm{f}}$, the assumption of spherical growth is reasonably justified [17]. In addition, it is assumed that very potent nucleating particles with a log-normal size distribution are present in the isothermal melt, and therefore the free growth criterion can be directly applied in the analysis. 
Solute contents have a strong influence on the solidification process of an isothermal melt through growth restriction [9]. The growth restriction factor $Q$ is usually taken as a quantitative measure of such an effect. The higher the $Q$ is, the lower the crystal growth velocity, and in turn the less latent heat is released from the growing crystals. Therefore, for alloys with higher $Q$, recalescence occurs at a lower temperature, providing a larger undercooling (larger $\Delta T_{\text {re }}$ ) for more particles to be active for nucleation. The number of active particles increases rapidly with the undercooling in the melt under the assumption of a log-normal distribution of particle size. The growth of these active particles eventually leads to recalescence. After recalescence, the undercooling required for the remaining particles in the melt is larger than $\Delta T_{\text {re }}$, and these particles become redundant. The grain initiation is then stopped by the recalescence, and $\Delta T_{\text {re }}$ corresponds to the required undercooling for the smallest active particles in the melt. By assuming that there is no grain multiplication by dendrite fragmentation, the grain density $N_{\mathrm{g}}$ should be equal to the active particle density $N_{\mathrm{a}}$, and then the total number of active particles determines the average grain size $\bar{l}$. At the same time, the accumulated solute ahead of the growing crystals reduces the undercooling available for grain initiation in the diffusional zone. Therefore grain initiation occurs outside the diffusion zone of the growing crystals, where the available undercooling increases with $Q$. Fig. 5 shows schematic cooling curves for the alloys with low and high $Q$. To sum up, the increase in solute content reduces velocity of crystal growth, increases the total undercooling achievable before recalescence. This in turn increases the number density of active particles for nucleation, and reduces the grain size in the solidified microstructure.

\subsection{The analytical model}

At temperatures between $T_{1}$ and $T_{\mathrm{f}}$, the radius of the $i^{\text {th }}$ spherical crystal $\left(r_{\mathrm{i}}\right)$ at the time $t$ is given by $[9,35]:$

$$
r_{i}=\lambda Q t t^{\top \times 2}
$$

where $\lambda$ is given by the invariant size approximation [35]:

$$
\lambda=\left[\frac{-S}{2 \pi^{1 / 2}}\right]+\left[\frac{S^{2}}{4 \pi}-S\right]^{1 / 2}
$$

and

$$
S=-\frac{2 \Lambda T-\Delta T_{\mathrm{c}}^{\mathrm{i}}-}{(-1) h C_{0}-\Delta T-\Delta T_{\mathrm{c}}^{\mathrm{i}} \iota}
$$

where $\Delta T$ is the total undercooling and $\Delta T_{\mathrm{c}}{ }^{\mathrm{i}}$ is the curvature undercooling of the $i^{\text {th }}$ crystal.

$\Delta T$ can be expressed as $\Delta T=\Delta T_{\mathrm{s}}+\Delta T_{\mathrm{c}}+\Delta T_{\mathrm{k}}$, where $\Delta T_{\mathrm{k}}$ is the kinetic undercooling. $\Delta T_{\mathrm{k}}$ may be neglected in comparison with $\Delta T_{\mathrm{s}}$ [9], and then $\Delta T_{\mathrm{s}}=\Delta T-\Delta T_{\mathrm{c}}$. For very potent nucleating particles, $\Delta T$ is very small, usually a few tenths of a degree. Therefore, one has $\left|m C_{0}\right| » \Delta T-\Delta T_{\mathrm{c}}$, and $|S| \ll 1$ from equation (9). Both equations (8) and (9) can be simplified as:

$$
\lambda=\left[\frac{-S}{2 \pi^{1 / 2}}\right]+\left[S^{\pi / 2}=\left[S^{\pi / 2}\right.\right.
$$

and

$$
S=-\frac{2\left(T-\Delta T_{\mathrm{c}}^{\mathrm{i}}\right)}{\uparrow-1 m C_{0}}=-\frac{2\left(T-\Delta T_{\mathrm{c}}^{\mathrm{i}}\right.}{Q} .
$$

Substituting equation (11) into (10), one obtains:

$$
\lambda=\left[\frac{2\left(T-\Delta T_{\mathrm{c}}^{\mathrm{i}}\right.}{Q}\right]^{1 / 2} .
$$


Differentiating equation (7) with respect to time gives the growth rate of the $i^{\text {th }}$ crystal as:

$$
V_{\mathrm{i}}=\frac{d r_{\mathrm{i}}}{d t}=\frac{1}{2} \frac{\lambda D^{1 / 2}}{t^{1 / 2}}
$$

Rearranging equation (7) gives:

$$
t=\frac{r_{\mathrm{i}}^{2}}{\lambda^{2} D} \text {. }
$$

Substituting equations (12) and (14) into (13) and rearranging, one has:

$$
r_{\mathrm{i}}=\frac{D\left(T-\Delta T_{\mathrm{c}}^{\mathrm{i}}-\right.}{V_{\mathrm{i}} Q} .
$$

The volume fraction of the solid at the moment of recalescence is given by:

$$
f_{\mathrm{sr}}=\sum_{i=1}^{N_{\mathrm{a}}} \frac{4}{3} \pi r_{\mathrm{i}}^{3} .
$$

For simplicity, it is assumed that the radius of growing crystals is identical at $\Delta T_{\mathrm{re}}$, and $f_{\mathrm{sr}}$ of the identical $N_{\mathrm{a}}$ crystals is the same as that of the actual growing crystals. Thus, equation (16) becomes:

$$
f_{\mathrm{sr}}=\frac{4}{3} \pi r_{\mathrm{e}}^{3} N_{\mathrm{a}}
$$

where $r_{\mathrm{e}}$ is the equivalent radius of the growing crystals, which is defined as the radius of $N_{\mathrm{a}}$ monosized crystals providing a solid fraction of $f_{\mathrm{sr}}$. These identical crystals have the same curvature undercooling $\Delta \bar{T}_{\mathrm{c}}$ according to equation (4), where $\Delta T_{\mathrm{fg}}=\Delta \bar{T}_{\mathrm{c}}$, and hence the same growth rate $\bar{V}$. Equation (15) becomes:

$$
r_{\mathrm{e}}=\frac{D \Lambda T_{\mathrm{re}}-\Delta \bar{T}_{\mathrm{c}}}{\bar{V} Q} .
$$

Rearranging equation (17) gives:

$$
N_{\mathrm{a}}=\frac{3 f_{\mathrm{sr}}}{4 \pi r_{\mathrm{e}}^{3}} \text {. }
$$

When recalescence occurs, $N_{\mathrm{a}}=N_{\mathrm{g}} . N_{\mathrm{g}}$ is linked with the average grain size $\bar{l}$ through the following equation [17]:

$$
N_{\mathrm{g}}=\frac{0.5}{\bar{l}^{3}} \text {. }
$$

One eventually obtains:

$$
\bar{l}=\left(\frac{2 \pi}{3 f_{\mathrm{sr}}}\right)^{1 / 3} r_{\mathrm{e}} .
$$

Substituting equation (18) into (21) produces:

$$
\bar{l}=\left(\frac{2 \pi}{3 f_{\mathrm{sr}}}\right)^{1 / 3} \frac{D \Delta T_{\mathrm{re}}-\Delta \bar{T}_{\mathrm{c}}}{\bar{V} Q} .
$$

Equation (22) describes theoretically the effect of solute on the grain size produced under isothermal solidification conditions due to growth restriction of solute $(Q)$ at the $\mathrm{S} / \mathrm{L}$ interface. In addition to $Q$, the key parameters affecting grain size are $f_{\mathrm{sr}}, \Delta T_{\mathrm{re}}, \Delta \bar{T}_{\mathrm{c}}$ and $\bar{V}$, which are all functions of $Q$, and can all be evaluated using the free growth criterion based on the procedures described in our previous paper [28]. Thus, the grain size can be calculated from equation (22) with $f_{\text {sr }}, \Delta T_{\text {re }}, \Delta \bar{T}_{\mathrm{c}}, \bar{V}, Q$ and $D$ as input parameters. The calculated grain sizes for AZ91D alloy and 
commercial-purity (CP) aluminium under different solidification conditions are compared with the experimental results [26,28] in Table 4. It is clear that the predicted gain sizes are in good agreement with the experimental results, suggesting that this analytical model can be used to predict the grain size.

However, $f_{\mathrm{sr}}, \Delta \bar{T}_{\mathrm{c}}$ and $\bar{V}$, as input parameters for grain size prediction, can not be determined by experiment, limiting the predicting power of Equation (22). It is desirable to simplify Equation (22), and this can be achieved by analysing the dependence of such parameters on the growth restriction factor $Q$.

For potent particles, $\Delta T_{\text {re }}$ is considerably small. $\Delta T_{\text {re }}$ can be obtained from the measurement of the cooling curves. On the other hand, it is not possible to obtain $\Delta \bar{T}_{\mathrm{c}}$ from an experimental observation. $\Delta \bar{T}_{\mathrm{c}}$ can be estimated according to equation (4) for $r_{\mathrm{e}}$ when the grain initiation is stopped, and the calculated $\Delta \bar{T}_{\mathrm{c}}$ is listed in Table 2 for $\mathrm{Mg}$ and $\mathrm{Al}$ alloys. It can be seen that $\Delta \bar{T}_{\mathrm{c}}$ is relatively small, compared to $\Delta T_{\text {re }}$, and can be neglected in most cases. Thus equation (22) can be simplified as:

$$
\bar{l}=\left(\frac{2 \pi}{3 f_{\mathrm{sr}}}\right)^{1 / 3} \frac{D \Delta T_{\mathrm{re}}}{\bar{V} Q} .
$$

According to the present model, the grain size is proportional to $f_{\mathrm{sr}}^{-1 / 3}$. Generally, $f_{\mathrm{sr}}$ is a function of $Q, \dot{T}$, the Gibbs-Thomson parameter $\Gamma$ and physical conditions of the grain refining particles. The theoretical calculations based on the free growth model [26] revealed that $f_{\mathrm{s}}$ increases with $Q, \dot{T}$ and the addition level of the grain refiner. For example, $f_{\text {sr }}$ increases from $1.5 \times 10^{-4}$ to $6.4 \times 10^{-4}$ while the cooling rate increases from $0.22 \mathrm{~K} / \mathrm{s}$ to $3.5 \mathrm{~K} / \mathrm{s}$ for AZ91 alloy melts with intensive melt shearing [28]. $f_{\text {sr }}$ increases from $1.5 \times 10^{-4}$ for $Q=2.21$ to $2.2 \times 10^{-4}$ for $Q=4.42$ in the CP-Al melt with the addition of 1p.p.t. Al-5Ti-1B [26]. Table 2 lists the values of $f_{\mathrm{sr}}$ for the alloys with different chemical compositions and solidification conditions. It can be seen that the variation of $f_{\text {sr }}$ is relatively small for typical casting conditions.

$\bar{V}$ can be estimated from an analysis of the experimental data. Ignoring $\Delta \bar{T}_{\mathrm{c}}$, rearranging equation (18) gives:

$$
\bar{V}=\frac{D \Delta T_{\mathrm{re}}}{r_{\mathrm{e}} Q} .
$$

The calculation results indicate that $\bar{V}$ is strongly dependent on the cooling rate, and exhibits a slight variation for various alloys at a given cooling rate. For example, at a cooling rate of $3.5 \mathrm{~K} / \mathrm{s}$ $\bar{V}$ is $0.05 \mathrm{~mm} / \mathrm{s}$ for $\mathrm{CP}$ Al with the addition of 1 p.p.t. Al-5Ti-1B, and $0.02 \sim 0.04 \mathrm{~mm} / \mathrm{s}$ in the compositional range of $0.1 \sim 9 \%$ for $\mathrm{Mg}_{1-\mathrm{x}} \mathrm{Al}_{\mathrm{x}}$ alloys. However, $\bar{V}$ decreases dramatically to about $0.00075 \mathrm{~mm} / \mathrm{s}$ for AZ91 alloy at a cooling rate of about $0.2 \mathrm{~K} / \mathrm{s}$.

Now let us analyse the dependence of $f_{\mathrm{sr}}, \Delta T_{\text {re }}$ and $\bar{V}$ on $Q$ by taking the Mg-Al alloy system as an example. Figs. 6(a)-(c) show the $f_{\text {sr }}, \Delta T_{\text {re }}$ and $\bar{V}$ evaluated from the experimental grain size data plotted against $1 / Q$. It is interesting to find that $f_{\text {sr }}, \Delta T_{\text {re }}$ and $\bar{V}$ are all closely related to $1 / Q$, and their dependence on $1 / Q$ can be described mathematically by a power law relationship as given in Fig. 6. By inserting such power law dependence on $1 / Q$ into equation (23), one obtains a much simplified version of equation (23): 


$$
\bar{l}=K\left(\frac{1}{Q}\right)^{1 / 3}
$$

where $K$ is a constant, which is independent of alloy composition, but strongly dependent on alloy systems, solidification conditions and the physical nature of nucleating particles, such as number density, size and size distribution. For a given alloy system and nucleating particles, $K$ represents the combined effect of potency and efficiency of the nucleating system. The smaller the value $K$ is, the higher the grain refining efficiency.

Fig. 7 shows a comparison between experimental data from the literature [36] and the prediction from equation (25) for the Al-Si and Al-Zn systems. The good agreement in Fig. 7 suggests that $(1 / Q)^{1 / 3}$ accounts for the solute effect more effectively than a simple linear relationship. In addition, it is found that the grain size of the majority of Al-based wrought alloys solidified under the TP-1 condition [22] can be well described as a function of $(1 / Q)^{1 / 3}$ with $K=0.58$, as clearly demonstrated in Fig. 8.

\section{Discussion}

Grain refinement through enhancing heterogeneous nucleation requires highly potent nucleating particles with a high efficiency. However, the terms of potency and efficiency have been used in the literature in a rather confusing way. To clear the confusion, we have recently provided the definition of both terms according to the epitaxial nucleation model [37]. Nucleating potency is defined as the degree of the perfection of the lattice matching at the solid/substrate interface during heterogeneous nucleation and the better the lattice match, the higher the nucleation potency will be. Therefore, nucleating potency is an inherent property of a given nucleating system, which consists of the substrate, the solid and the liquid composition. By contrast, nucleating efficiency refers to the effectiveness of a given type of solid particles (e.g., $\mathrm{TiB}_{2}$ ) with specific physical characteristics and solidification conditions, such as number density, size, size distribution of the nucleating substrates, as well as cooling rate. For a given nucleating system, nucleation potency is fixed but nucleation efficiency can be changed by modifying the physical characteristics of the nucleating particles and/or changing the solidification conditions. Based on such definitions, we can analyse the effect of a solute on heterogeneous nucleation.

Solute atoms affect the potency of a given nucleating system. According to the expitaxial nucleation theory [37], solute atoms may segregate at the liquid/substrate interface at temperatures above the alloy liquidus if this reduces the interfacial energy. The segregated solute atoms modify the crystallographic mismatch between the substrate and the solid phase during the nucleation process by either changing the lattice parameter of the solid solution or by formation of an intermetallic phase. If this modification reduces the misfit it will increase the potency of the nucleation system, (enhancing heterogeneous nucleation); if the solute atoms at the liquid/substrate interface enlarge the misfit it will decrease the potency and poison the heterogeneous nucleation.

Solute atoms also affect nucleation efficiency. Based on the free growth criterion, the large nucleating particles start to grow as soon as the available undercooling reaches their $\Delta T_{\mathrm{fg}}$ in the isothermal melt. If the partition coefficient $k<1$, the growing new crystal will reject solute to the S/L interface, causing constitutional undercooling and growth restriction. As discussed previously in section 2, for an isothermal melt, constitutional undercooling will reduce the total undercooling, and will not be able to activate particles in the diffusion zone for heterogeneous nucleation. However, as demonstrated in section 3, growth restriction will allow more particles with a suitable size to be active for heterogeneous nucleation before recalescence, resulting in enhanced nucleation efficiency and a finer grain size in the solidified microstructure. 
Based on the above analysis, Equation (23) has only taken into account the effect of solute on the nucleation efficiency by assuming all the nucleating particles are highly potent. Therefore, equation (23) can only be used to elucidate the effect of a solute on grain refinement through growth restriction, and its predicting power should not be overstated. However, although largely empirical, Equation (25) can account for the overall effect of solute on both potency and efficiency, and can be used to predict the grain size for a given nucleating system once the constant $K$ has been evaluated from the experimental data for the system, as has been demonstrated in Figs. 7 and 8.

In addition, the present model (equations (23) and (25)) provides a new understanding of the solute effect on grain refinement. Firstly, Equations (23) and (25) limit well. They give $l \rightarrow 0$ when $Q \rightarrow \infty$. This means that an alloy with infinitely large growth restriction will give an infinitely small grain size. This is in contrast to the linear relationship (equation (3)), which predicts that $l \rightarrow a$ when $Q \rightarrow \infty$, being clearly unphysical. Secondly, the $1 / 3$ power law offers a more accurate description of the experimental data available in the literature than the linear relationship $[1,4,22,24]$. The plots of grain size against $1 / Q$ based on the experimental data in the literature frequently exhibit a deviation from the linear approximation at the high $Q$ end for both binary and multi-component systems $[1,22,24]$. In contrast, the $1 / 3$ power law (equation (25)) offers a better agreement with the experimental data, particularly at the high $Q$ end of the plot. Thirdly, the model parameter $K$ can be easily determined by a few careful experiments for a given alloy system solidifying under similar experimental conditions. This allows an easy assessment of the alloy composition on the grain size once $K$ is evaluated. Finally, the model parameter $K$ can be used as a direct measurement of the grain refining efficiency of a given nucleating system in comparison with other nucleating systems. The smaller the $K$ is, the higher the grain refining efficiency is.

Accurate prediction of grain size in an as-cast microstructure is very difficult if at all possible. Grain size is affected by both nucleation and growth processes. During growth after completion of nucleation, grain multiplication through dendrite fragmentation may take place under certain solidification conditions and result in a refined microstructure. Even if grain multiplication is eliminated and the grain size is completely determined by the nucleation process, there are still many factors affecting nucleation, and therefore grain size. It is well understood that heterogeneous nucleation is affected by alloy composition, the chemical and physical natures of the nucleating particles and the exact solidification conditions. Alloy composition affects both the potency of nucleating particles through adsorption at the liquid/particle interface and the efficiency through growth restriction; The crystal structure of the nucleating particles affects the misfit and therefore potency; the physical nature of the particles, such as number density, size and size distribution determine the nucleation efficiency; the solidification conditions, such as cooling rate and temperature gradient in the melt, also have a strong influence on nucleation efficiency. It seems impossible to take simultaneously all such variables into consideration in one single analytical model. Consequently, analytical modelling of grain refinement can only be focused on understanding the nucleation mechanism rather than an accurate prediction of grain size.

\section{Summary}

The effects of solute content on grain refinement in isothermal melts have been analysed. In an isothermal melt, an increase of $Q$ restricts the growth of previously initiated grains, and results in larger undercooling being available for grain initiation on smaller particles until the grain initiation is stopped by the recalescence, leading to a finer grain size after solidification. On the other hand, the accumulated solute elements in the diffusional zone of the growing crystal actually reduce the undercooling available for grain initiation, and the grain initiation should occur only on the particles outside the diffusional zone. 
An analytical model has been developed to predict the grain size solidified under an isothermal condition. This analytical model revealed that the relationship between $\bar{l}$ and $1 / Q$ can be described as $\bar{l}=\mathbb{Q} \pi / 3 f_{\mathrm{sr}}{ }^{\top / 3} D \Delta T_{\mathrm{re}} \bar{V} Q$, where $f_{\mathrm{sr}}, \Delta T_{\mathrm{re}}$ and $\bar{V}$ are all closely related to $1 / Q$. As a result, the grain size can be simply related to $(1 / Q)^{1 / 3}$ through a single parameter $K$. Comparison with the experimental data from the literature has confirmed that the $1 / 3$ power law describes the experimental grain size more accurately than the conventional linear relationship.

\section{Acknowledgements}

The authors wish to thank Dr. Y. Zuo for discussion about experimental grain size data of the MgAl binary alloys, and Prof. B. Ralph for his reading of the manuscript. EPSRC is gratefully acknowledged for providing financial support under grant EP/H026177/1.

\section{References:}

[1] StJohn DH, Qian M, Easton MA, Hildebrand Z. Metall Mater Trans A 2005;36:1669.

[2] McCartney DG. Int Mater Rev 1989;34:247.

[3] Murty BS, Kori SA, Chakraborty M. Int Mater Rev 2002;47:3.

[4] Atamanenko TV, Eskin DG, Zhang L, Katgerman L. Metall Mater Trans A 2010;41:2056.

[5] Bunn AM, Schumacher P, Kearns MA, Boothroyd CB, Greer AL. Mater Sci Technol $1999 ; 15: 1115$.

[6] Hunt JD. Mater Sci Engi 1984;65:75.

[7] Gäumann M, Trivedi R, Kurz W. Mater Sci Engi A 1997;226-228;763.

[8] Tarshis CA, Walker JL, Rutter JW. Metall Trans 1971;2:2589.

[9] Maxwell I, Hellawell A. Acta Metall 1975;23:229.

[10] Easton MA, StJohn DH. Metall Mater Trans A 1999;30:1613.

[11] Easton MA, StJohn DH. In: Angier J, (Eds.). Light Metals. Warrendale (PA): TMS; 2001, pp. 927-33.

[12] Chai G, Bäckerud L, Arnberg L. Mater Sci Technol 1995;11:1099.

[13] Johnsson M. Z Metallkd 1994;85:781.

[14] Easton MA, StJohn DH. Acta Mater 2001;49:1867.

[15] Desnain P, Fautrelle Y, Meyer J-L, Riquet J-P, Durand F. Acta Mater Metall 1990;38:1513.

[16] Jones GP, Pearson J. Metall Trans B 1976;7:223.

[17] Greer AL, Bunn AM, Tronche A, Evans PV, Bristow DJ. Acta Mater 2000;48:2823.

[18] Backerud L, Johnsson M. In: Hale W, (Eds.). Light metals. Warrendale (PA): TMS; 1996. p. 679-85.

[19] Kearns MA, Cooper PS. Mater Sci Technol 1997;13:650.

[20] StJohn DH, Cao P, Qian M, Easton MA. Adv Eng Mater 2007;9:739.

[21] Bermingham MJ, McDonald SD, StJohn DH, Dargusch MS. J Alloys Comp 2009;481:L20.

[22] Easton M, StJohn D. Metall Mater Trans A 2005;36:1911.

[23] Winegard WC, Chalmers B. Trans Am Soc Metals 1954;46:1214.

[24] Qian M, Cao P, Easton MA, McDonald SD, StJohn DH. Acta Mater 2010;58:3262.

[25] Quested TE, Greer AL. Acta Mater 2005;53:4643.

[26] Quested TE, Greer AL. Acta Mater 2004;52:3859.

[27] Günther R, Hartig Ch, Bormann R. Acta Mater 2006;54:5591.

[28] Men H, Jiang B, Fan Z. Acta Mater 2010;58:6526.

[29] Avedesian M, Baker H. Magnesium and magnesium alloys. Materials Park (OH): ASM; 1999. p. 231-33.

[30] Eustathopoulos N, Coudurier L, Joud JC, Desré P. J Cryst Growth 1976;33:105.

[31] Brandes EA. Smithells Metals Reference Book, sixth ed. London: Butterworths; 1983, pp. 8-114-1.

[32] Eremenko VN, Natanzon VN, Titov VP. Fizika Khim Mekh Mater 1978;14:3. 
[33] Massalski TB. Binary Alloy Phase Diagrams, second ed. Materials Park: ASM; 1990.

[34] Mullins WW, Sekerka RF. J Appl Phys 1963;34:323.

[35] Aaron HB, Fainstein D, Kotler GR. J Appl Phys 1970;41:4405.

[36] Spittle JA, Sadli S. Mater Sci Technol 1995;11:533.

[37] Fan Z. To be submitted. 


\section{Tables:}

Table 1 Physical properties used in the calculations.

\begin{tabular}{lcc}
\hline \multicolumn{1}{c}{ Alloys } & AZ91 & CP Al \\
\hline Gibbs-Thomson parameter, $\Gamma(\mathrm{K} \mathrm{m})$ & $1.48 \times 10^{-7}[29]$ & $1.42 \times 10^{-7}[30,31]$ \\
Solute diffusivity in melt, $D\left(\mathrm{~m}^{2} / \mathrm{s}\right)$ & $2.7 \times 10^{-9}[29]$ & $2.52 \times 10^{-9}[32]$ \\
Liquidus slope, $m(\mathrm{~K} / \mathrm{wt} \%)$ & $-6.87^{[1]}$ & $-25.63^{[33]}$ \\
Equilibrium partition coefficient, $k$ & $0.37^{[1]}$ & $7^{[33]}$ \\
\hline
\end{tabular}

Table 2 Summary of experimental and calculated results for four sets of alloy and processing conditions in the isothermal melts.

\begin{tabular}{lcccc}
\hline \multicolumn{1}{c}{ Alloys } & AZ91 & AZ91 & CP Al & Al-0.4wt\%Ti \\
\hline Process condition & Melt shearing & Melt shearing & 1 p.p.t. Al-5Ti-1B & - \\
$\dot{T}(\mathrm{~K} / \mathrm{s})$ & 0.22 & 3.5 & 3.5 & 1.5 \\
$f_{\mathrm{sr}}$ & $1.5 \times 10^{-4}[28]$ & $6.4 \times 10^{-4 \mathrm{~b}}$ & $1.5 \times 10^{-4}[26]$ & $\sim 10^{-4}[9]$ \\
$\bar{l}(\mathrm{~mm})$ & $1.21^{[28]}$ & $0.178^{\mathrm{b}}$ & $0.103^{[26]}$ & $0.203^{[9]}$ \\
$N_{\mathrm{g}}\left(\mathrm{mm}^{-3}\right)$ & 0.35 & 88.7 & 458 & 60 \\
$C_{\mathrm{s}}(\mathrm{wt} \%)$ & 3.33 & 3.33 & 0.09 & 2.8 \\
$C_{0}(\mathrm{wt} \%)$ & 9.0 & 9.0 & 0.012 & 0.4 \\
$C_{\mathrm{i}}(\%)$ & $9.04^{\mathrm{a}}$ & $9.01^{\mathrm{a}}$ & 0.0111 & 0.3966 \\
$\Delta T_{\mathrm{s}}(\mathrm{K})$ & 0.28 & 0.07 & 0.023 & 0.086 \\
$\Delta \bar{T}_{\mathrm{c}}(\mathrm{K})$ & 0.0063 & 0.025 & 0.066 & 0.0098 \\
$\Delta T_{\mathrm{re}}(\mathrm{K})$ & 0.62 & 1.08 & 0.2 & 1.45 \\
\hline
\end{tabular}

${ }^{a}$ Only diffusion of $\mathrm{Al}$ atoms is considered.

${ }^{\mathrm{b}}$ Calculated using free growth modelling with the approaches available in Ref. [28]

Table 3 The variation of $\Delta T_{\mathrm{s}}$ and $\Delta T_{\text {re }}$ at recalescence against the cooling rate for the intensively sheared AZ91 alloy melt

\begin{tabular}{lccccc}
\hline$\dot{T}(\mathrm{~K} / \mathrm{s})$ & 0.1 & 1 & 10 & 100 & 1000 \\
\hline$f_{\text {sr }}$ & $1.4 \times 10^{-4}$ & $2.5 \times 10^{-4}$ & $1.05 \times 10^{-3}$ & $1.52 \times 10^{-3}$ & $1.72 \times 10^{-3}$ \\
$\bar{l}(\mathrm{~mm})$ & 1.0887 & 0.267 & 0.0603 & 0.0206 & 0.00774 \\
$N_{\mathrm{g}}\left(\mathrm{mm}^{-3}\right)$ & 0.387 & 26.3 & 2280 & 57196 & 1078319 \\
$C_{\mathrm{s}}(\mathrm{wt} \%)$ & 3.33 & 3.33 & 3.33 & 3.3 & 3.3 \\
$C_{0}(\mathrm{wt} \%)$ & 9 & 9 & 9 & 9 & 9 \\
$C_{\mathrm{i}}(\%)$ & 9.016 & 9.006 & 9.005 & 9.005 & 9.006 \\
$\Delta T_{\mathrm{s}}(\mathrm{K})$ & 0.11 & 0.04 & 0.03 & 0.03 & 0.04 \\
$\Delta T_{\mathrm{re}}(\mathrm{K})$ & 0.63 & 0.88 & 1.32 & 1.87 & 2.75 \\
\hline
\end{tabular}

Table 4 The grain sizes calculated with the present model.

\begin{tabular}{lccc}
\hline & AZ91 & AZ91 & CP Al \\
\hline Grain refinement & Melt & Melt & 1 p.p.t. Al- \\
Shearing & shearing & 5Ti-1B \\
condifitions & Isothermal & Isothermal & Isothermal \\
$\dot{T}(\mathrm{~K} / \mathrm{s})$ & 0.22 & 3.5 & 3.5 \\
$\Delta T_{\mathrm{re}}(\mathrm{K})$ & $0.62^{[28]}$ & $1.08^{\mathrm{a}}$ & $0.2^{[26]}$ \\
$f_{\text {sr }}$ & $1.5 \times 10^{-4}[28]$ & $6.4 \times 10^{-4} \mathrm{a}$ & $1.5 \times 10^{-4}[26]$ \\
$\bar{l}(\mathrm{~mm})$ (this model) & 1.51 & 0.163 & 0.146 \\
$\bar{l}(\mathrm{~mm})$ (previous data) & $1.21^{[28]}$ & $0.178^{\text {a }}$ & $0.103^{[26]}$ \\
\hline
\end{tabular}


${ }^{\text {a }}$ Calculated using free growth modelling with the approaches available in Ref. [28] 


\section{Figure captions:}

Fig. 1 Measured cooling curve for the AZ91 alloy melt with intensive melt shearing. The cooling curve exhibits pronounced recalescence, in which $T_{\mathrm{f}}$ corresponds to the temperature at the moment of recalescence and $T_{\mathrm{g}}$ the thermal plateau after recalescence. The maximum undercooling $\Delta T_{\mathrm{re}}$ at recalescence is estimated by the value of $T_{\mathrm{g}}-T_{\mathrm{f}}$. The measured cooling rate near the liquidus $T_{1}$ is $0.22 \mathrm{~K} / \mathrm{s}$, and $\Delta T_{\mathrm{re}}$ is $0.62 \mathrm{~K}$.

Fig. 2 An illustration of a growing grain $(\mathrm{S})$ in an isothermal melt $(\mathrm{L}) . r$ is the radius of the growing grain, and $D / V$ is the characteristic width of the concentration profile in the liquid ahead of the solid/liquid (S/L) interface. This assumes that the solute atoms rejected by the solid diffuse only into the region between $r$ and $r+D / V$.

Fig. 3 Schematic representation of the concentration profile. $C_{\mathrm{s}}$ and $C_{\mathrm{i}}$ are the solute concentrations of the solid and liquid, respectively, at the S/L interface. For simplicity, the solute content in the bulk solid is considered to be uniform and approximate to $C_{\mathrm{s}}=k C_{0}$ at the initial stage of solidification. At this stage the solute profile in the liquid can be well approximated by a straight line: $C=C_{\mathrm{i}}-\frac{C_{\mathrm{i}}-C_{0}}{D / V} x$.

Fig. 4 Schematic representation of the liquidus $\left(T_{1}\right)$ and local temperature $\left(T_{\mathrm{lc}}\right)$ profiles ahead of a growing crystal in an isothermal melt. $\Delta T$ is the local thermal undercooling, $\Delta T_{\mathrm{s}}$ the solutal undercooling, and $\Delta T_{\mathrm{kc}}$ the sum of kinetic and curvature undercoolings. $\Delta T_{\mathrm{s}}$ reduced the available undercooling for nucleation of the remaining particles ahead of a growing crystal.

Fig. 5 Schematic cooling curves for the isothermal melts with low and high $Q$, respectively. In the melt with low $Q$, the largest particle starts grain initiation at the point A of the cooling curve at which the temperature is denoted by $T_{\mathrm{s}}$, and the grain initiation is finished at $\mathrm{B}\left(T_{\mathrm{f}}\right)$ due to the occurrence of recalescence. The high $Q$ implies a slower latent heat release and larger $\Delta T_{\text {re, }}$ as indicated by the cooling curve with high $Q$, and therefore leads to a finer grain size.

Fig. 6 The plots of (a) the solid fraction $f_{\text {sr }}$, (b) $\Delta T_{\text {re }}$ and (c) average growth velocity $\bar{V}$ at recalescence against $1 / Q$ for intensively sheared $\mathrm{Mg}$-Al binary alloy melts, calculated using the approaches available in Ref. [28]. It can be seen that the values of $f_{\text {sr }}, \Delta T_{\text {re }}$ and $\bar{V}$ are related to $1 / Q$. The fitted curves give a power of $-0.71,-0.71$ and 0.14 to $1 / Q$ for $f_{\mathrm{sr}}, \Delta T_{\mathrm{re}}$ and $\bar{V}$, respectively.

Fig. 7 Comparison of experimentally determined grain size data against $1 / Q$ for binary Al-Zn and Al-Si binary alloys [36] with the prediction from equation (25). The fitted (solid) curves with equation (25) show a good agreement with the experimental data.

Fig. 8 Comparison of experimentally determined grain size data against $1 / Q$ for $\mathrm{Al}$ wrought alloys with additions of 0.005 pct $\mathrm{TiB}_{2}$ at various titanium levels [22] with the prediction (solid curve) from equation (25). This indicates that the experimental data can be well described by the relationship of $\bar{l} \sim(1 / Q)^{1 / 3}$. 
Figures:

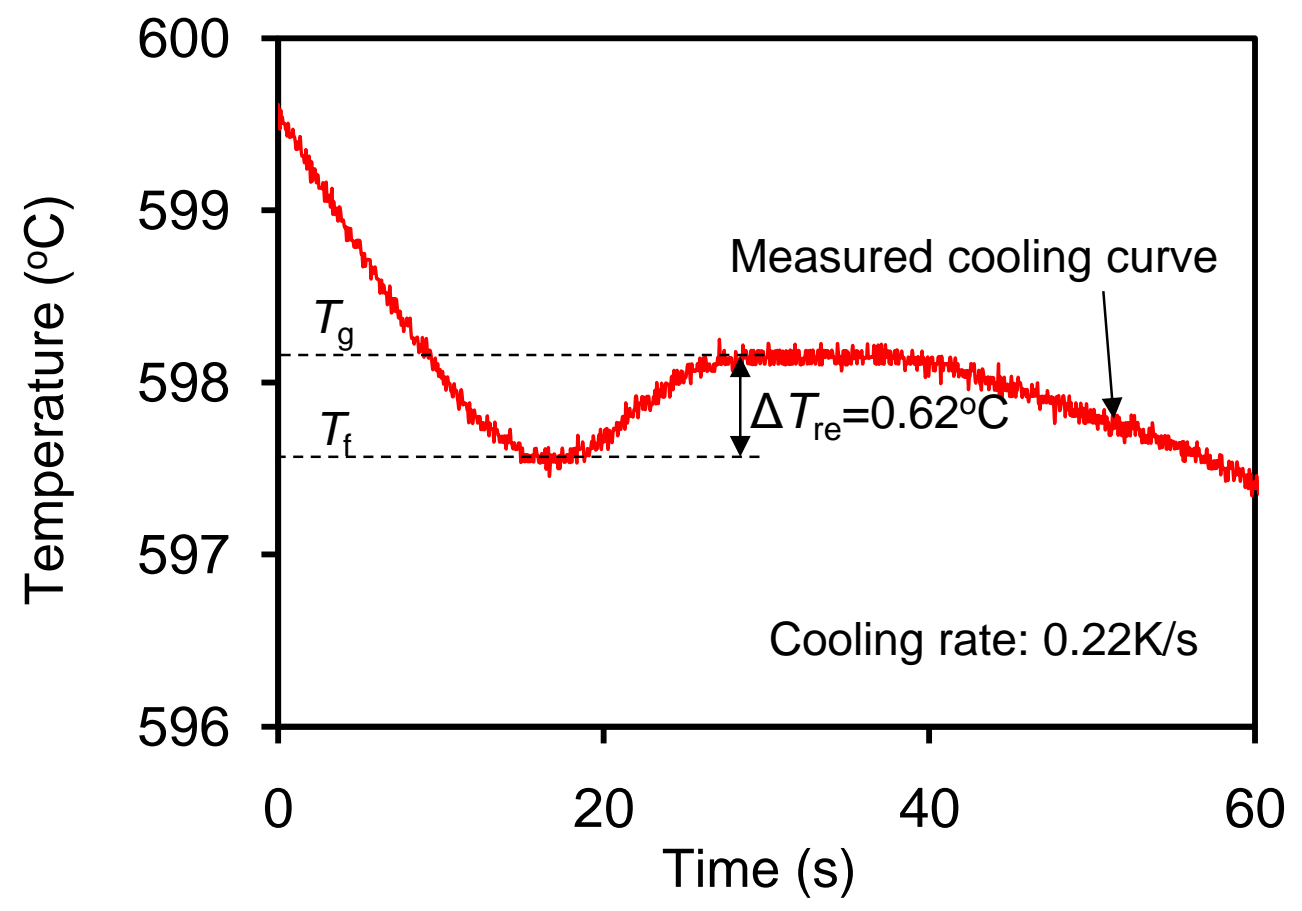

Fig. 1 


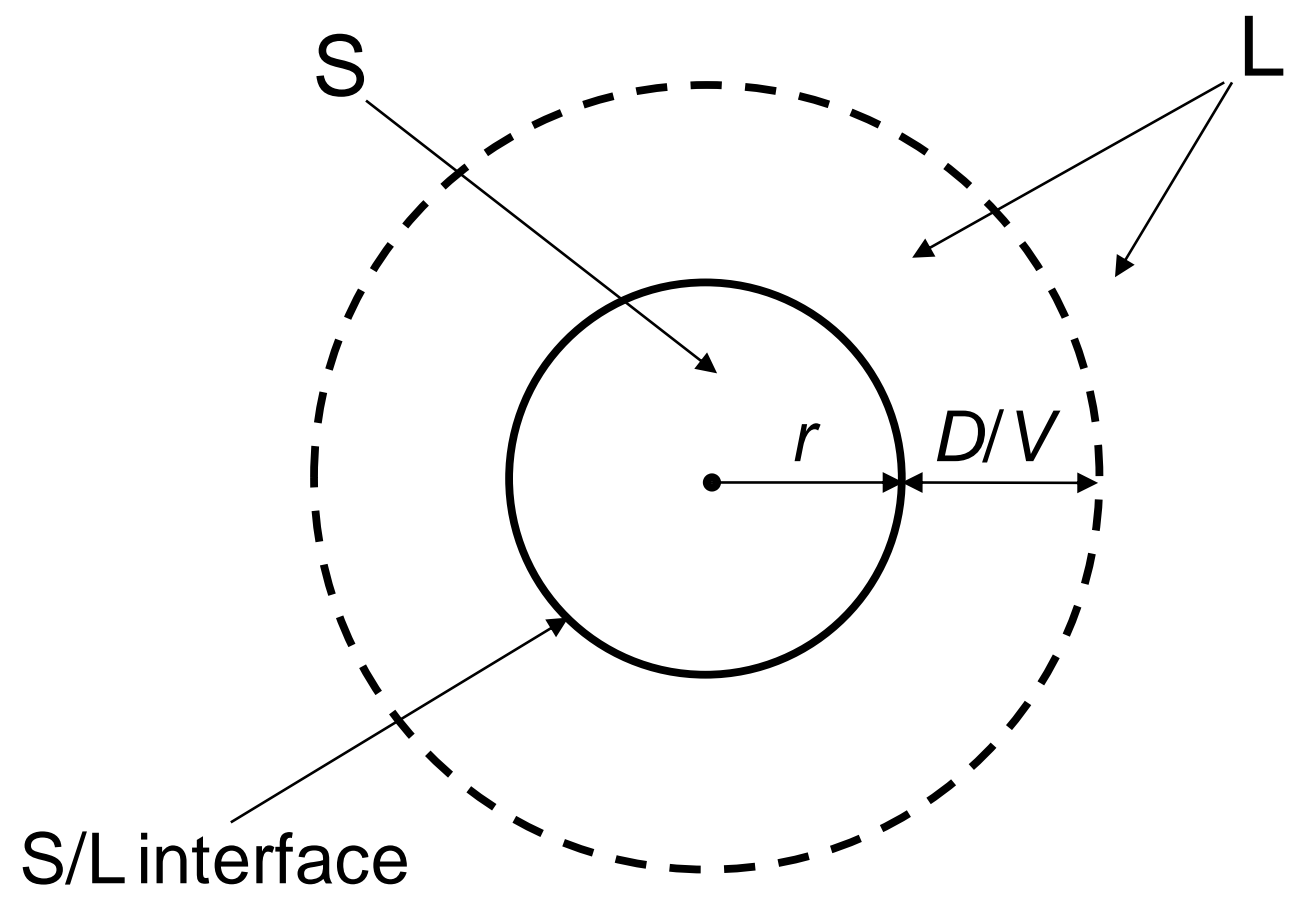

Fig. 2 


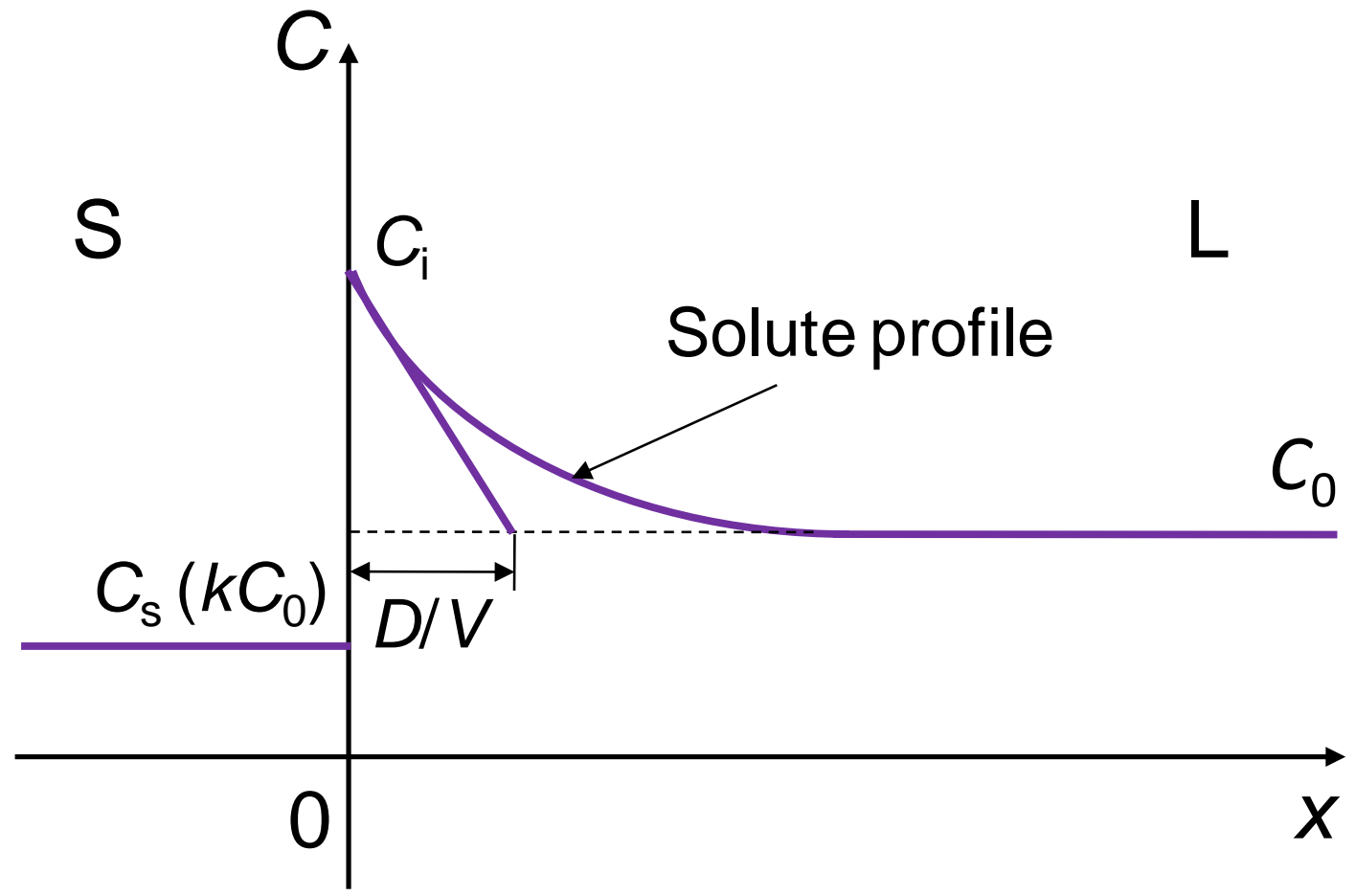

Fig. 3 


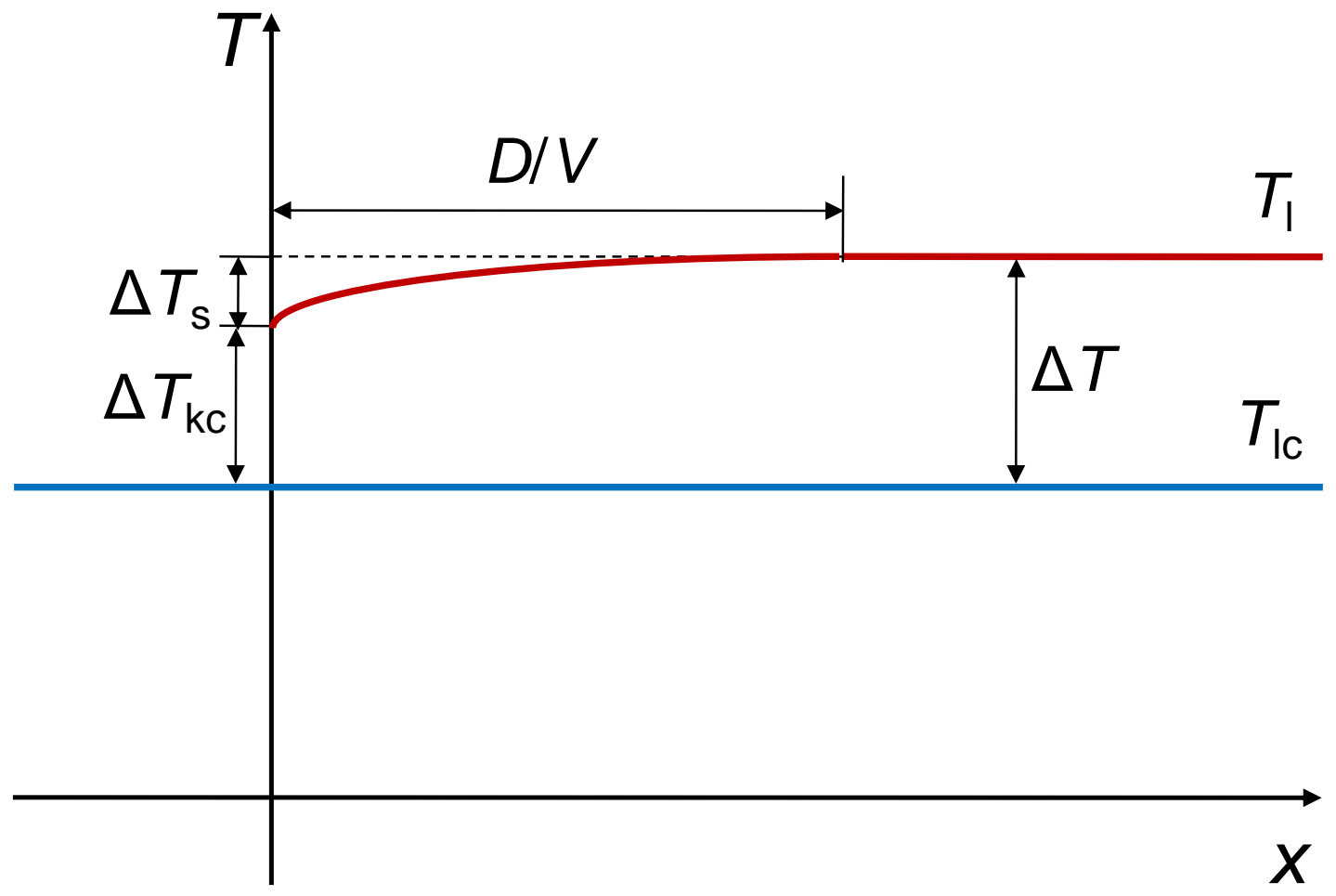

Fig. 4 


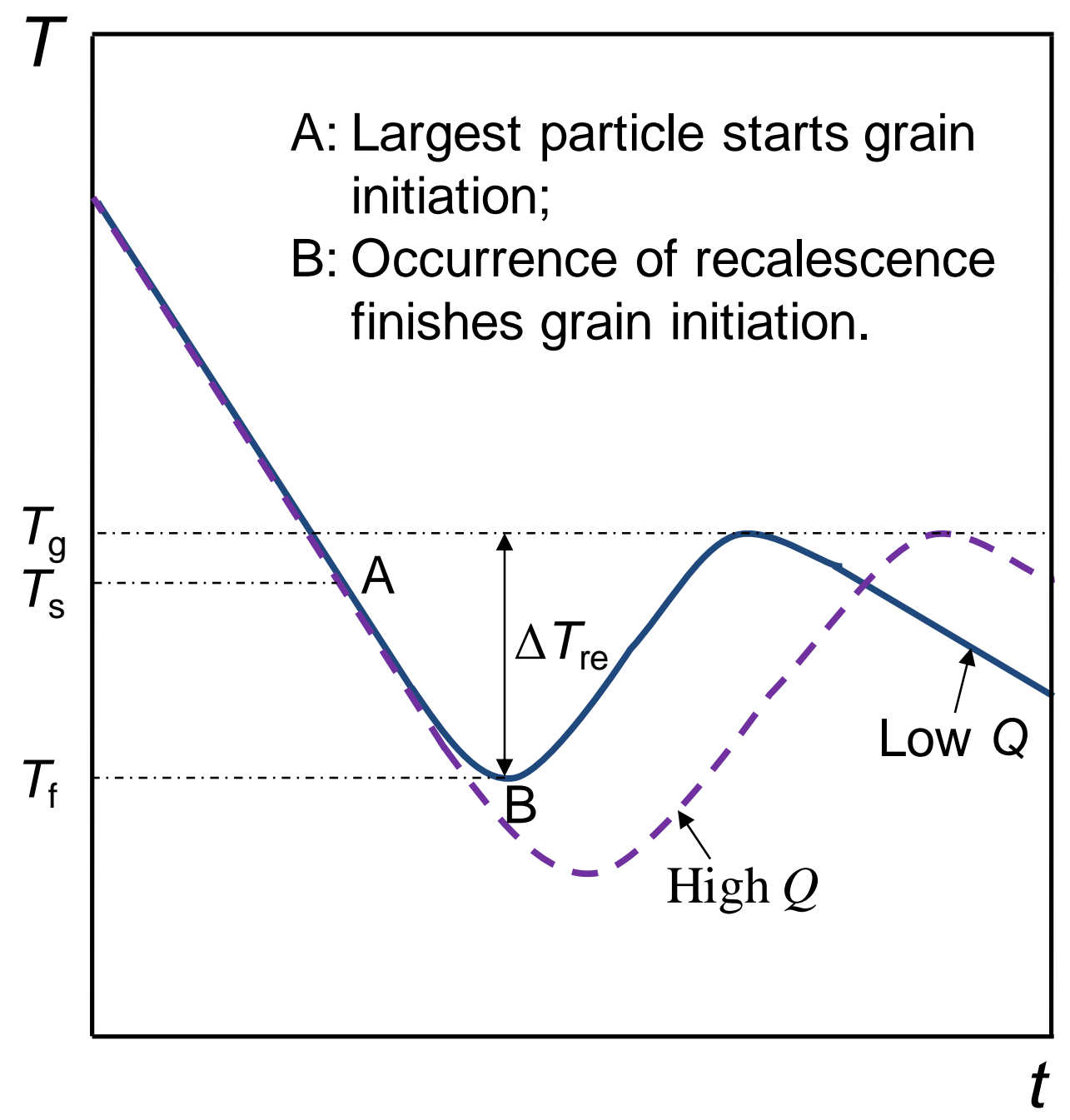

Fig. 5 


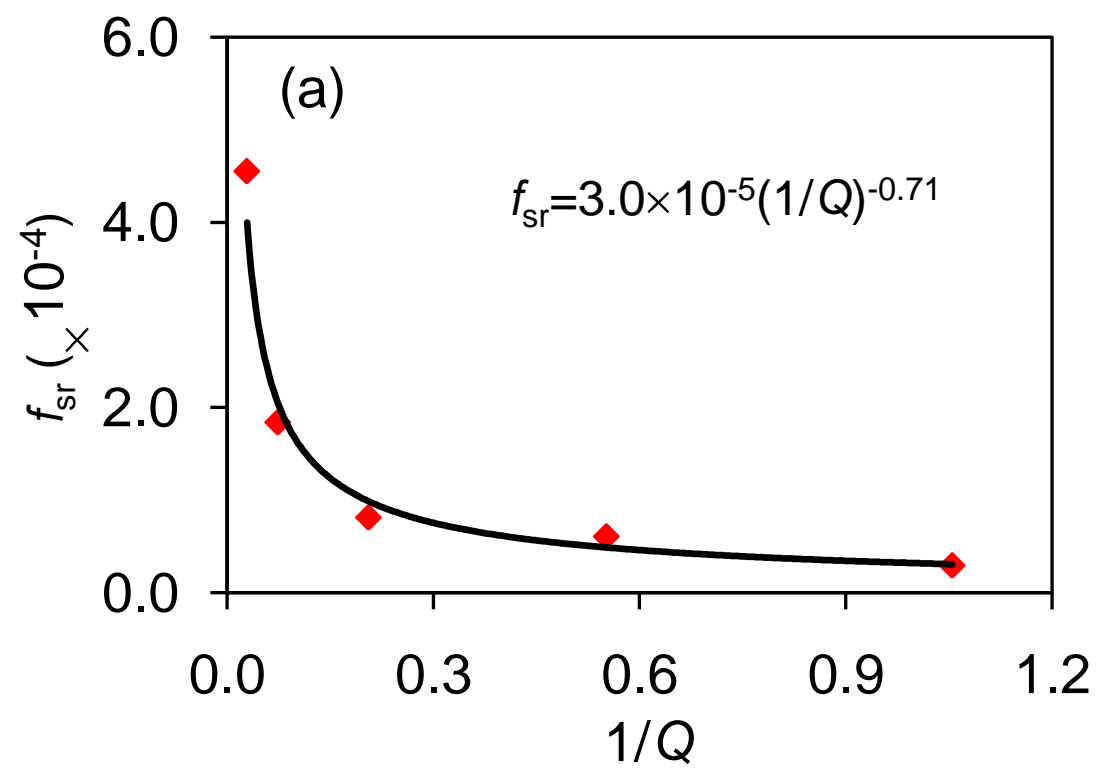

Fig. 6(a)

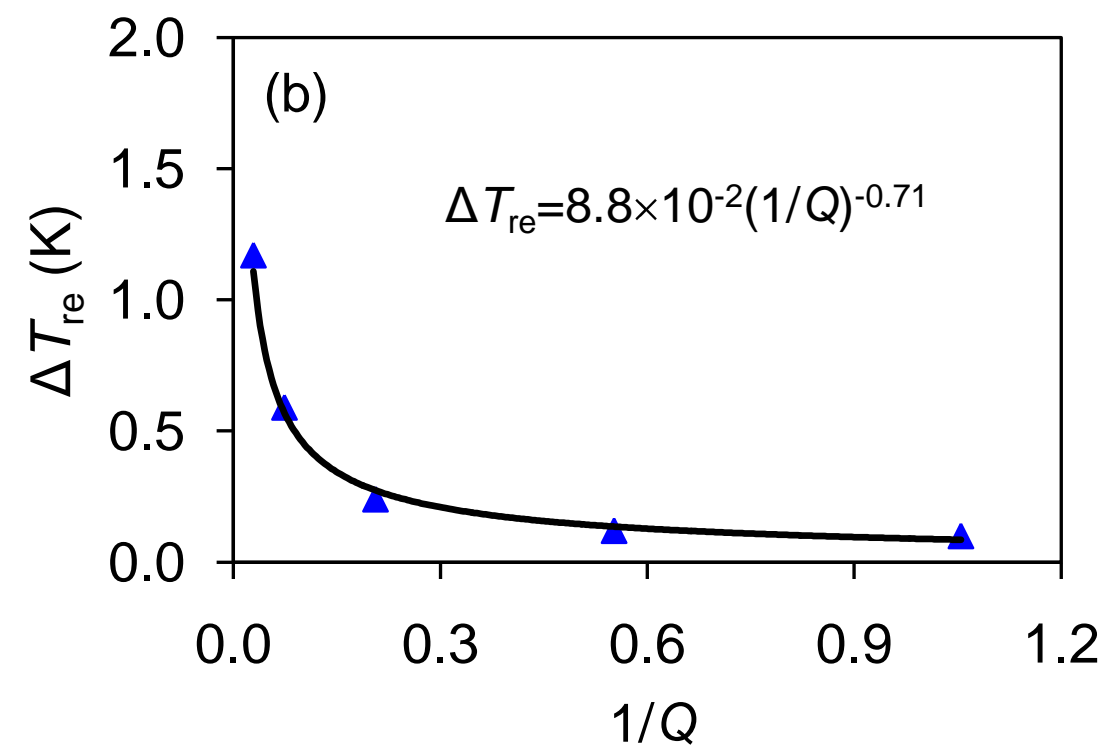

Fig. 6(b) 


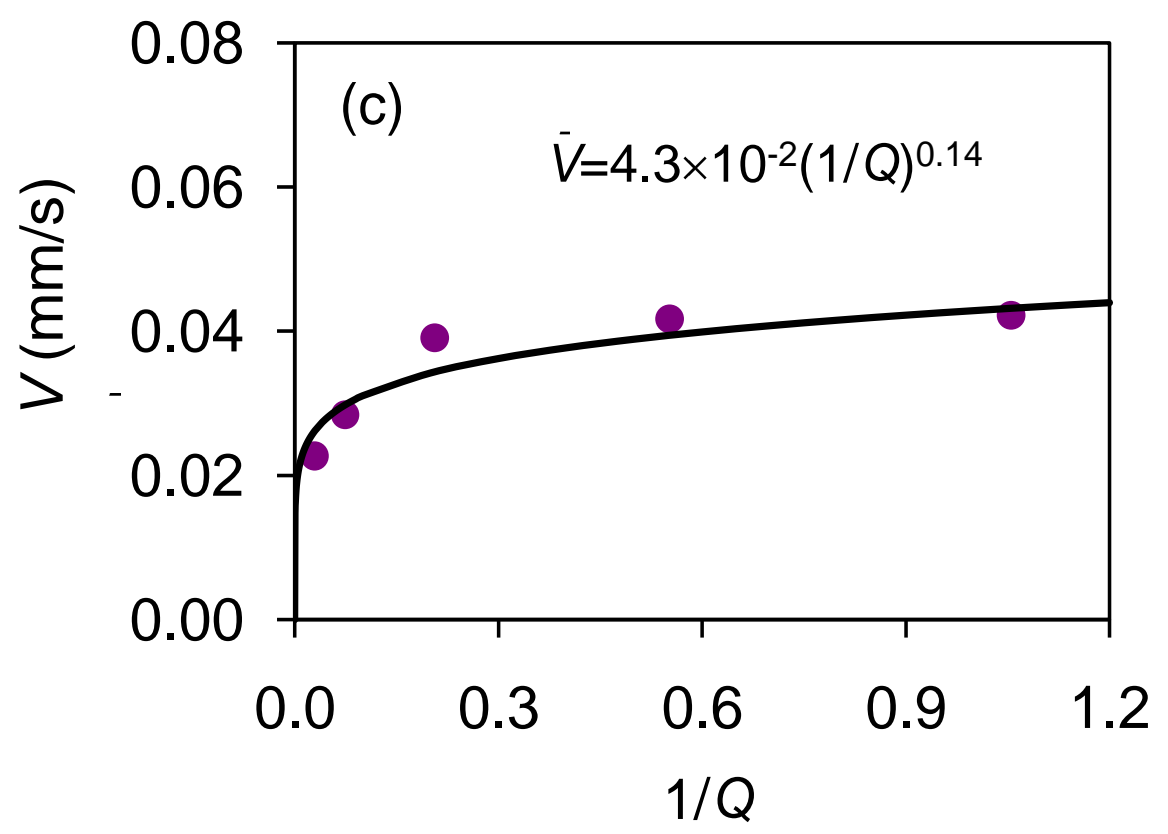

Fig. 6(c) 


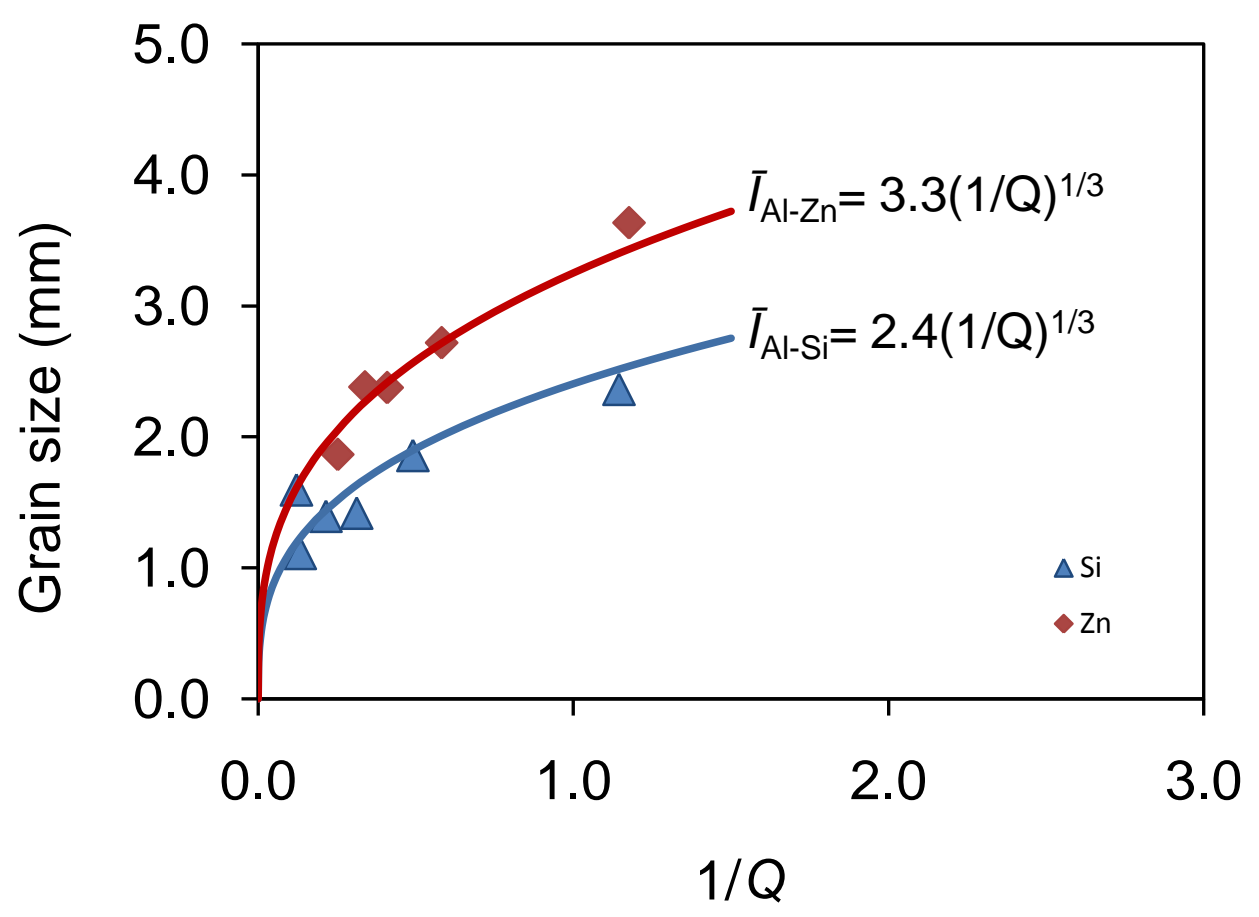

Fig. 7 


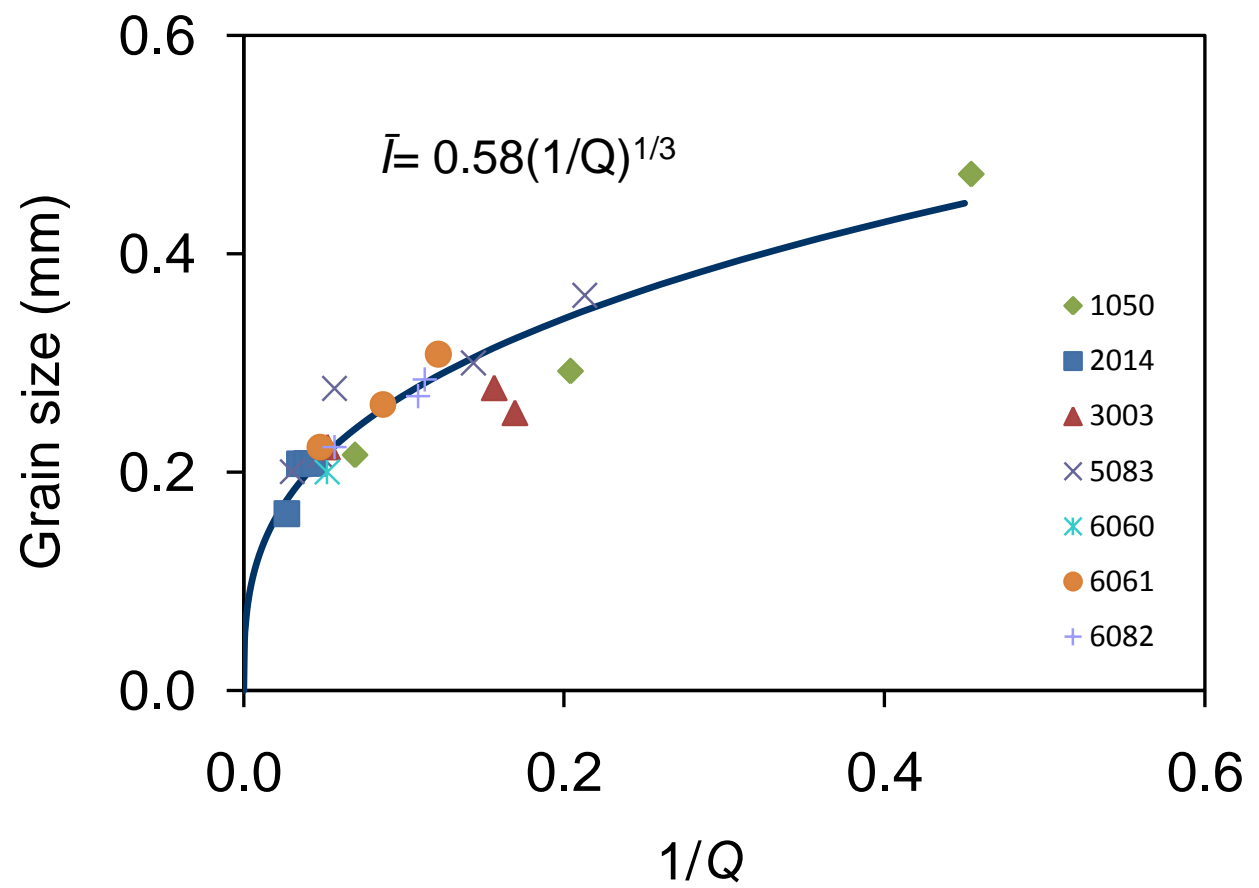

Fig. 8 